\title{
IN-ORBIT STATUS OF THE HIPPARCOS ASTROMETRY MISSION
}

\author{
M.A.C. PERRYMAN \\ Astrophysics Division \\ ESTEC, Postbus 299 \\ 2200 AG Noordwijk \\ The Netherlands
}

\begin{abstract}
The Hipparcos satellite was successfully launched by Ariane 4 flight 33, from French Guyana, on 8 August 1989. However, the apogee boost motor, designed to place the satellite into its intended geostationary orbit, failed to function, and the satellite was left in a highly elliptical transfer orbit. After a perigee raising manoeuvre, using the hydrazine propulsion system, the orbital parameters are approximately: apogee $35900 \mathrm{~km}$, perigee $526 \mathrm{~km}$, eccentricity 0.72 , inclination $6.8^{\circ}$, orbital period $10^{\mathrm{h}} 40^{\mathrm{m}}$.

Operational procedures have had to be revised during the first weeks of satellite operations, due to the non-nominal orbit. Commissioning and calibrations have taken longer than foreseen because of the operational difficulties and observational overheads, but routine observations commenced on 27 November. The essential differences between the nominal and the present missions are that observations can only be conducted for about 30-40 per cent of real time in the present mission, and the fact that the actual lifetime of the mission is still rather uncertain, and possibly somewhat shorter than anticipated. This paper gives a summary of the present satellite status, measurement results obtained so far, and in indication of the scientific goals that may be achievable with the present mission.
\end{abstract}

\section{Introduction}

Hipparcos, the European Space Agency's astrometry mission, has been fully described in the ESA publication SP-1111 (June 1989). Launched by an Ariane 4 from French Guyana on 8 August 1989, the failure of the apogee boost motor to place the satellite in its planned geostationary orbit appeared at first to strongly degrade the expected scientific return of the mission. However, apart from the failure of the apogee boost motor, the satellite behaviour has been entirely nominal, and the degradation of the solar arrays due to the particle radiation environment (the principal cause of the expected reduced mission lifetime) has not been as significant as had originally been predicted.

The perigee altitude was raised to $500 \mathrm{~km}$ on 7-8 September, the solar arrays and fill-in antenna were successfully deployed on 12 September, the baffles were opened on 25 September, the first star mapper measurements were made on 26 September, and 'initial star pattern recognition' (the term given to the initial determination of the satellite's three-axis attitude), with the satellite in sun-pointing mode, was successfully completed on 
8 October. All six on-board detectors, the prime and redundant image dissector tubes, and the prime and redundant pairs of star mapper photomultipliers, are functioning nominally, as are the on-board mechanisms-the shutters, the switching mirror, and the refocusing mechanism.

All further instrumental calibrations were completed by 25 November, and the nominal data collection phase of the mission started on 27 November.

\section{Orbital Parameters and Mission Lifetime}

The satellite required hydrazine as reaction control fuel only for the spinning phase in transfer and drift orbit. The tanks were filled to their full capability of $32 \mathrm{~kg}$, and a surplus of $26 \mathrm{~kg}$, corresponding to a $\Delta V$ of about $47 \mathrm{~ms}^{-1}$, was available for orbit manoeuvres. This was sufficient to raise the perigee to about $500 \mathrm{~km}$ to avoid atmospheric drag and atomic oxygen problems.

It was also possible to adjust the orbital period to a multiple of the great circle scan period. Such a scan-synchronous orbit allows the minimisation of occultations and avoids earth albedo light into the payload. The revised orbit maneouvres were completed on 18 September 1989, and the orbit characteristics for that epoch are:

$\begin{array}{ll}\text { Perigee height } & 526 \mathrm{~km} \\ \text { Apogee height } & 35900 \mathrm{~km} \\ \text { Orbital period } & 640 \mathrm{~min} \\ \text { Eccentricity } & 0.72 \\ \text { Inclination } & 6.8^{\circ} \\ \text { Ascending node } & 105^{\circ} \\ \text { Argument of perigee } & 214^{\circ}\end{array}$

\section{Eclipses}

Long (> $60 \mathrm{~min}$ ) eclipses will occur in February/March 1990 and again in July 1990. The eclipse durations are not only longer than in the nominal orbit (100 min maximum, compared to $72 \mathrm{~min}$ for the nominal mission), but the ratio of the eclipse period to the orbital period becomes significantly larger. It is presently expected that the payload thermal control will have to be switched off during these intervals, probably making the resulting data unusable during this time interval.

The revised orbit results in an eclipse duration profile very different from that of a geostationary orbit. The eclipses will probably determine distinct operational phases:

(a) first operational phase ( 2 months) lasting from the beginning of end November 1989 , the planned completion date of payload calibration, to 2 February 1990 when the eclipse duration increases rapidly;

(b) hibernation phase ( 2 months) lasting from 2 February to 9 April 1990, the end of the first eclipse season;

(c) second operational phase (3 months) lasting from 9 April to 13 July 1990, the beginning of the second eclipse season;

(d) third operational phase (13 months) lasting from 13 July to mid August when the eclipse duration increases rapidly once again. 


\section{Ground Station Coverage}

In the nominal mission, the Odenwald ground station (FRG) would have been in continuous coverage with the satellite, and the total mission deadtime (due to occultations, calibrations, etc) would have amounted to less than 10 per cent. The present orbit, however, is covered by Odenwald for only 32 per cent of the time. The Perth and Kourou ground stations are included in the revised mission, resulting in about 81 per cent data acquisition time compared to full coverage. As a result of other operational and observational overheads, useful data collection occurs for about 30 per cent of the time.

However there are still non-visibility periods of more than 10 hours, a few times per week. It is under investigation to have a fourth ground station which would improve the coverage to 95 per cent and, more importantly, would reduce the non-visibility periods to less than 1 hour. The fraction of time for which useful data could be accumulated would also rise to about 50 per cent.

\section{The Satellite Performance}

\subsection{POWER BUDGET}

In the revised orbit the satellite power budget has to take into account three new aspects:

(1) degradation of solar cells due to increased dose from the radiation belts;

(2) temperature increase of the solar cells at perigee due to earth albedo light;

(3) worsened battery charge/discharge ratio due to the reduced orbital period and increased eclipse duration.

The effect of the solar array degradation due to the trapped high energy proton flux, is illustrated in Figure 1. Measurements of the in-orbit degradation, through measurements of the short-circuit current and the open-circuit voltage of the solar arrays, is presently in progress. Current lifetime prediction due to the solar array degradation is in the range 18-30 months. This prediction will be refined as the mission proceeds.

\subsection{THE ATTITUDE CONTROL AND DETERMINATION}

The attitude control and determination has to cope with three new problems:

(1) high disturbance torques at perigee;

(2) high star mapper rejection rates in the radiation belts;

(3) long non-visibility periods without reference stars.

5.2.1. High Disturbance Torques. The scientific measurements are based on an extremely smooth motion of the satellite leading to a control concept which leaves the satellite basically in free drift with occasional small thruster firings to keep the attitude within an allocated band around the nominal scanning law.

This concept is no longer valid around perigee due to the high disturbance torques caused by atmospheric drag, gravity gradient and magnetic torques. More frequent and more powerful thruster firings are required to counteract the high disturbance torques. As a consequence below $10000 \mathrm{~km}$ altitude the control parameters have to be changed to provide a shorter 'look ahead' time and to allow for a longer thruster on-times. This not only reduces the value of the scientific measurements but also causes a problem for a hardwired back- 


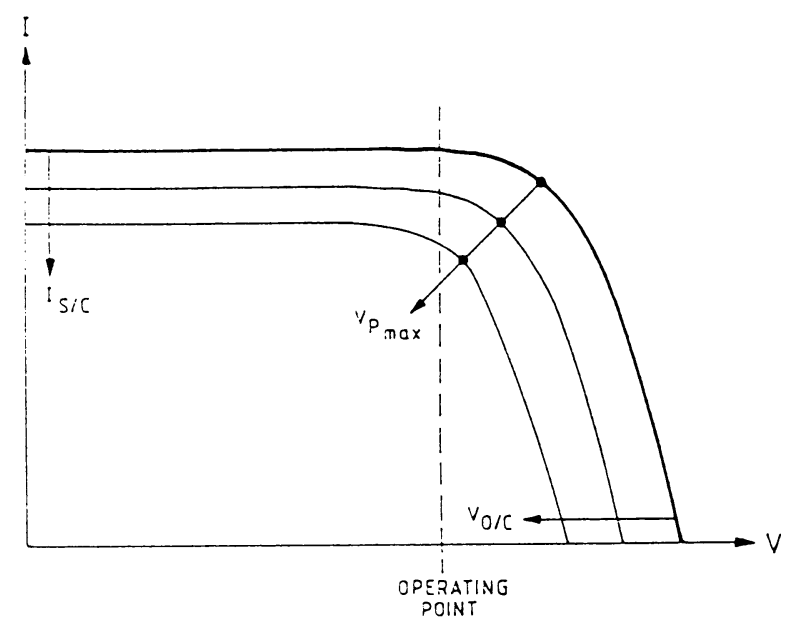

Figure 1. Radiation effects on the solar cells. The effect of the degradation is to decrease the short-circuit current and open-circuit voltage, such that the voltage at maximum power $\left(V_{\mathrm{P} \max }\right)$ also decreases.

up function, referred to as the thrust impulse monitoring. The thrust impulse monitoring monitors the length and frequency of the thruster firings and switches automatically to the back-up branch of the attitude control subsystem if the hardwired thresholds are exceeded. For low altitude operations the thrust impulse monitoring therefore has to be disabled.

This new control concept has been already implemented with two control parameter sets controlled by time-tagged commands according to the predicted attitude. It has already worked successfully through many orbits. The cold gas consumption is only slightly higher than had been expected for the nominal mission.

5.2.2. High Star Mapper Rejection Rate. The background count rate of the star mapper increases drastically in the radiation belts. Consequently for a period of 2 to 3 hours around perigee all reference stars, except maybe very bright ones, will be rejected by the star mapper filtering process. Nevertheless, apart from those times affected by large solar flares, the attitude determination generally re-converges after perigee passages.

5.2.3. Long Non-Visibility Periods. The Hipparcos system requires a continuous uplink of the programme star file which contains the programme stars being measured and the reference stars needed to update the attitude determination and to guide the satellite along the nominal scanning law. The on-board programme star file buffer is sized to cover a time span of 1 to 2 hours. It is, however, insufficient to cover non-visibility periods of up to 12 hours. The solution envisaged is to use a special 'sparse programme star file' which contains only reference stars at time intervals just sufficient to support the attitude determination task. 


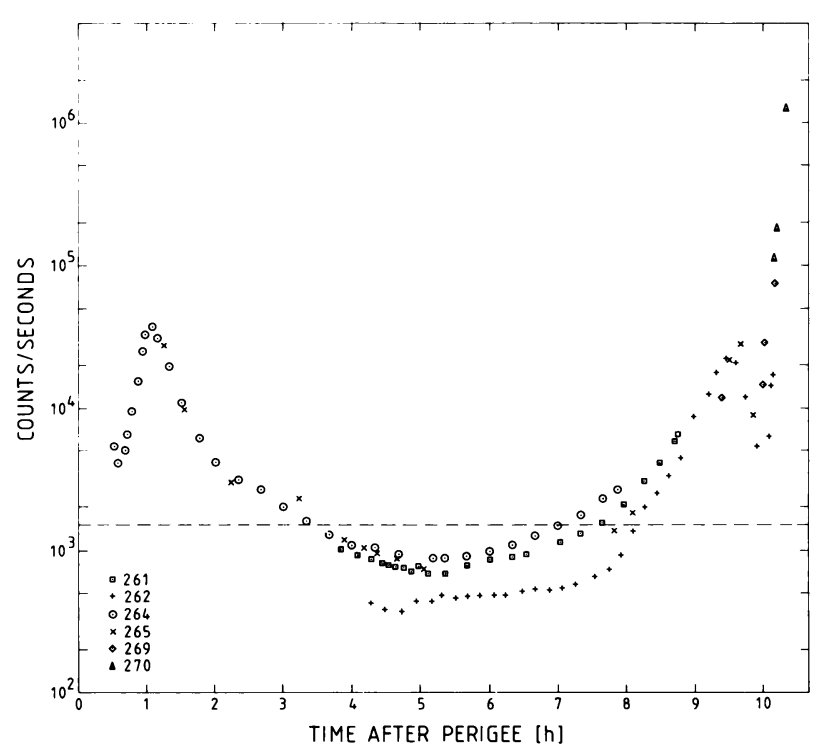

Figure 2. Photomultiplier tube background count rate. The numbers associated with the symbols indicate the day of the year 1989 .

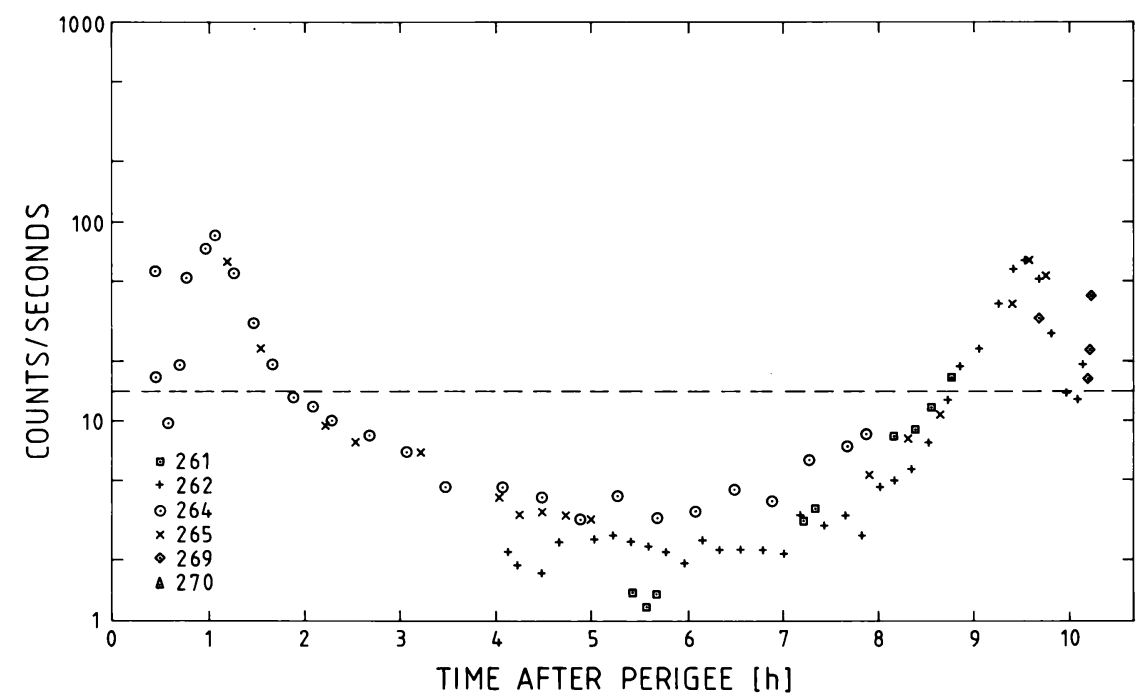

Figure 3. Image dissector tube background count rate. The numbers associated with the symbols indicate the day of the year 1989 . 


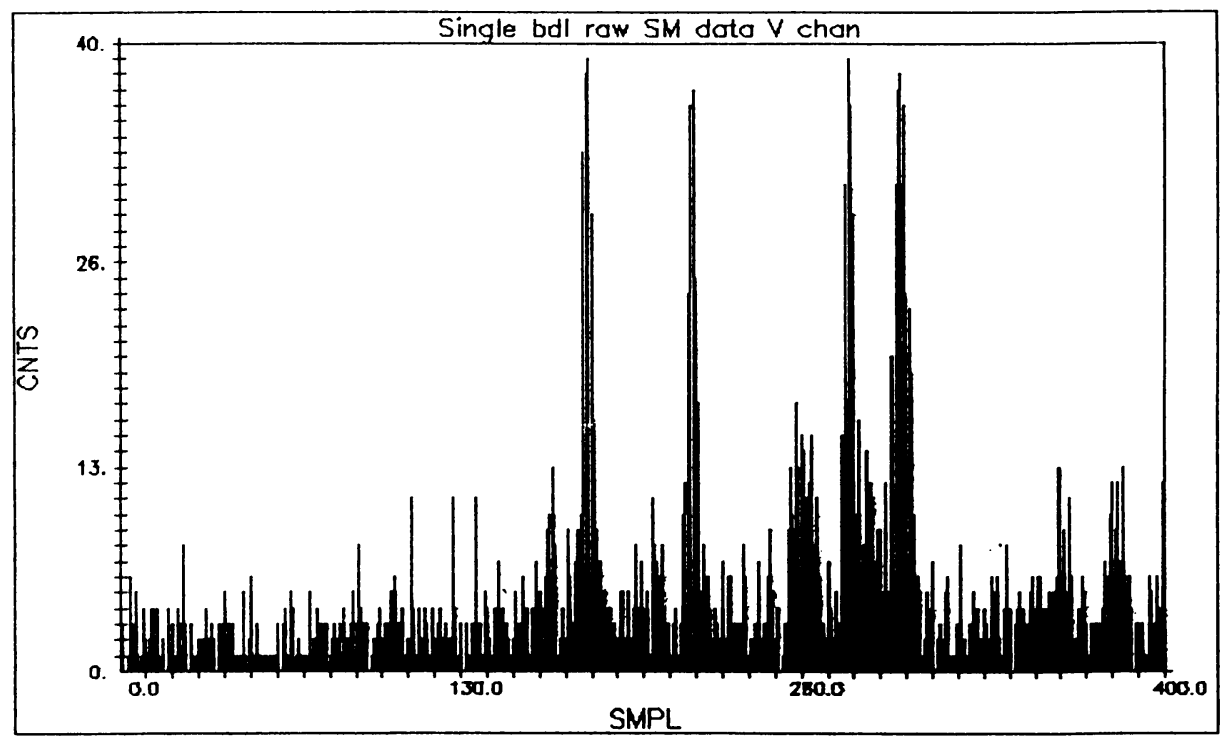

Figure 4. First results from the star mapper $V$ channel.

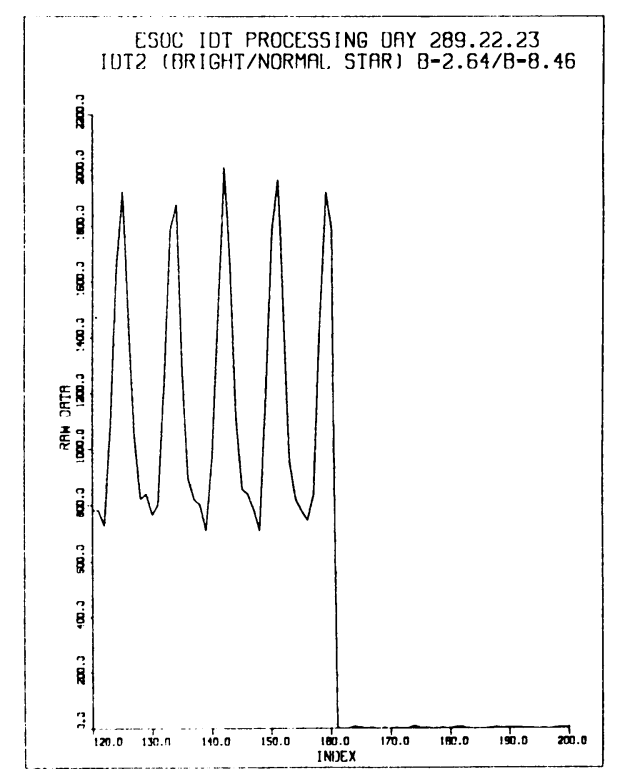

Figure 5. First results from the image dissector tube detector. The two stars observed are of magnitude 2.6 (at the left) and 8.5 (at the right, where the modulation is just visible). 


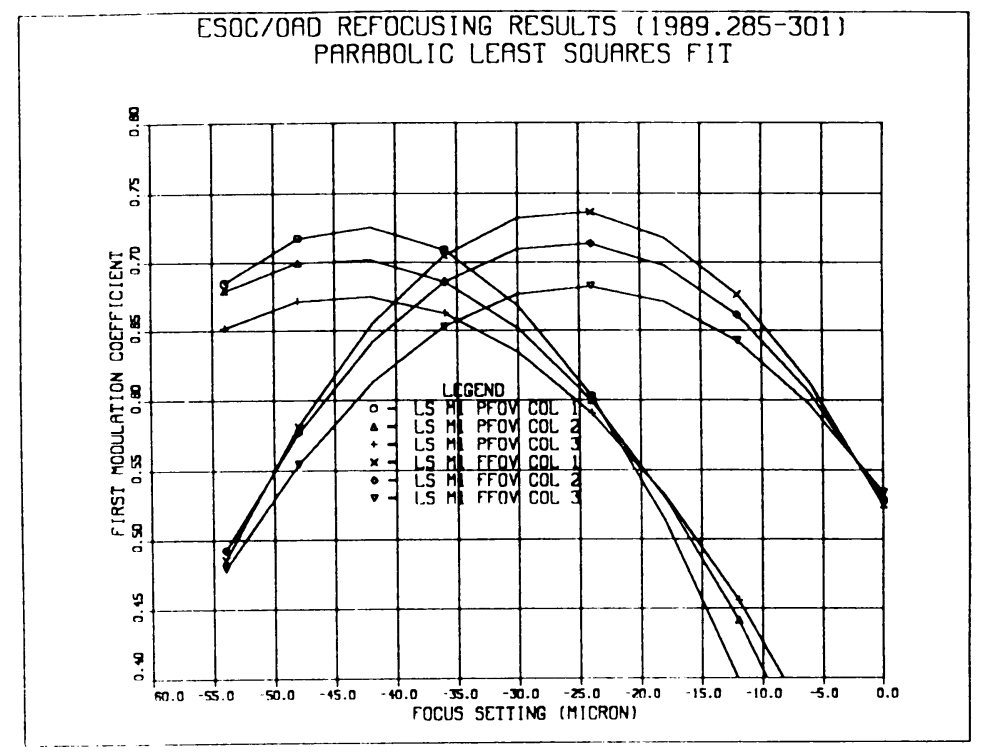

Figure 6. The effect of focusing on the modulation of the image dissector tube photon counts, as a function of the field of view and star colour. A good focus for both fields of view is achieved for a focus setting of -35 microns.

\section{The Payload Detectors}

The main payload related problem in the revised mission orbit is the increased background count rate due to Cerenkov and luminescence light caused by the high particle flux in the radiation belts. Some results are given in Figure 2 (background count rate of photomultiplier $B$ ) and Figure 3 (background count rate of the image dissector tube). The dotted lines indicate the background count rate assumed for accuracy analysis of the nominal mission. For the image dissector tube the background count rate most of the time is well below the nominal background level. In the 4 hour period around perigee (i.e. below an attitude of $23000 \mathrm{~km}$ ) the levels are less than a factor of 10 higher than nominal, and thus becomes comparable to straylight levels. It therefore can be expected that the limiting magnitude of observable stars is slightly reduced-from 12.5 to perhaps $12 \mathrm{mag}$ in this region. The situation for the photomultiplier $B$ is less favorable. The excess above the nominal background level is almost 1.5 orders of magnitudes for the outer belt and 3 orders of magnitude for the inner belt. Due to this high background the use of the star mapper for attitude determination within 3 hours around perigee is very doubtful.

The absolute background count rate levels are however well below levels affecting the detector hardware characteristics. It is therefore not necessary to switch off the detector high voltage supplies during radiation belt passages.

First results from the star mapper detector and the image dissector tube detector, showing the characteristic responses of the star transits, are shown in Figures 4 and 5 
Table 1. Expected accuracies for various durations of the revised mission. The table is based on a nominal operational capability of the the revised mission.

\begin{tabular}{lcccc}
\hline Mission Product & $\begin{array}{c}\text { Nominal } \\
\text { Mission } \\
(30 \text { months })\end{array}$ & \multicolumn{3}{c}{ Revised Mission Duration } \\
(months) & $(6)$ & $(18)$ & $(30)$ \\
\hline No. of stars & 120000 & 80000 & 110000 & 120000 \\
Observations per star & 80 & $0-10$ & 30 & 50 \\
Positions (arcsec) & 0.002 & 0.015 & 0.006 & 0.003 \\
Parallaxes (arcsec) & 0.002 & - & 0.006 & 0.004 \\
Proper motions (arcsec/yr) & 0.002 & - & 0.008 & 0.005 \\
\hline
\end{tabular}

respectively. The effect of focusing on the modulation of the image dissector tube photon counts, as a function of the field of view and star colour, is shown in Figure 6.

\section{Scientific Observations and Sky Coverage Uniformity}

The scanning law and the implementation of the observing programme do not have to be modified compared with the nominal mission, which means that the original Input Catalogue will still form the basis of the observing programme.

The number of observations for a particular star increases roughly in proportion to the mission lifetime. Sky coverage uniformity also improves with mission duration. However, the ability to decouple the determination of the positions, parallaxes and proper motions of the stars is also a function of the mission duration. In the nominal mission, a lifetime of at least 18 months was required to provide (a) full sky coverage, and (b) allow the astrometric parameter determination. Simulations by the Data Reduction Consortia have confirmed that, for the revised mission, a lifetime of at least 18 months is required for a fully self-consistent set of Hipparcos positions, parallaxes and proper motions to be obtained.

\section{Predicted Accuracies}

Estimates of the expected acuracy achievable by the present mission (and hence the scientific return) are critically dependent on the lifetime of the satellite. Present predictions indicate that, just as for the nominal mission, if the lifetime is less than 18 months then the scientific returns are strongly degraded compared with expectations-a substantial proportion of the scientific programmes would not be satisfied. However, a mission lifetime extending beyond 24 months improves the achievable accuracy, although the results will still represent some degradation with respect to original expectations. The assessment of the scientific impacts of this degradation are ongoing. A better indication of the scientific returns will be possible when the operational difficulties, which affect the total amount of useful data that can be acquired, have been fully evaluated. Table 1 provides an indication of the accuracies that may be achievable for various durations of the revised mission, on the assumptions that the operations can be conducted without significant loss of observing time, or without a significant degradation of the data quality. These assumptions will be confirmed over the coming weeks. 


\section{Discussion}

Editor's note: The oral paper was presented by E. Høg, who responded to the comments and remarks.

Miунмото: Prior to going to the discussion about the resolution, I would like to tell you that I, on behalf of IAU Commission 8, wrote a letter on 11 September to Prof. Lüst of ESA, in which I requested ESA to give earnest consideration to a new mission of HIPPARCOS as early as possible. Then, I received a reply of 22 September from Prof. Lüst, in which he states:

"I certainly anticipate activity in the scientific circles in Europe on the matter of Hipparcos 2 , especially among the scientists who will have to accept that their projects be delayed, but you can be assured of my determination to look at this issue in depth."

HøG: Let us take this as an encouragement.

MORRISON: Was any consideration given to changing the observing strategy in a limited mission: e.g., by limiting the observations to brighter stars?

Høg: These questions were considered at the first Science Team meeting one week after the launch. The conclusion was quickly reached that nothing could be gained by changes in the scanning law, input catalogue, or observation strategy.

RATNATUNGa: Could you please comment on the prospect of a second hIPPARcos and the continuity of observations with the current mission in the event of a decision for a replacement satellite which is so vital for astronomy?

HøG: I hope that all possible scientific observations will be continued with the present satellite for, say, 2 years, and I believe that most scientists will share this hope. Careful scientific arguments must of course be presented for such a continuation because the operational cost is far from negligible.

HuGHEs: Are the two new additonal downlink stations now operational?

Høg: The one in Australia is operational, but I am not certain about the one in Kourou in French Guiana.

\section{Note added in proof:}

Updated information on 1 February 1990: since this paper was accepted for publication, progress with the HIPPARCOS operations have continued to improve the overall mission outlook in three main areas:

(1) indications are now that a satellite lifetime of 30 months or more may now be achievable;

(2) the long eclipse durations are likely to have a much smaller scientific impact than originally expected;

(3) the fraction of useful scientific data acqusition is now in the range 55-60 per cent, and should increase further once the NASA Goldstone ground station is incorporated into the network during March 1990 . The first great circle reductions have been completed by the data reduction teams, and have confirmed the very high quality of the scientific data. If a mission lifetime of 36 months is reached, astrometric parameters close to the orginal 2 milliarcsec targets might still be achievable. 